\title{
Using Configural Frequency Analysis as a Person-centered Analytic Approach with Categorical Data
}

\author{
Mark Stemmler' and Jörg-Henrik Heine ${ }^{2}$
}

\begin{abstract}
Configural frequency analysis and log-linear modeling are presented as person-centered analytic approaches for the analysis of categorical or categorized data in multi-way contingency tables. Person-centered developmental psychology, based on the holistic interactionistic perspective of the Stockholm working group around David Magnusson and Lars Bergman, is briefly revisited. According to personcentered theory, systems or individuals are seen as a whole and as inseparable units; individuals are embedded and strongly interconnected with their context; the individual and the environment influence each other, and the individual is seen as an active agent or producer of his or her own development. Four models of configural frequency analysis are presented: (I) First-order configural frequency analysis, which is basically the analysis of a main effects log-linear model; (2) prediction configural frequency analysis, which defines one or more dependent variables; (3) two-group configural frequency analysis, which proposes that there is no association between discrimination variables and group membership; and (4) functional configural frequency analysis, which allows us to blank out certain outlier cells in order to test for the quasi-independence of the rest of the cross-table. The use of the open source Rpackage confreq for computational analysis is demonstrated. The advantages, as well as the limitations, of configural frequency analysis are discussed.
\end{abstract}

\section{Keywords}

Person-centered methods, configural frequency analysis, log-linear modeling, holistic interactionistic approach, organismic-systemic approach, R-package (open-source software), confreq

Configural frequency analysis (Krauth \& Lienert, 1973; von Eye, 2002) is a person-centered analytic approach for the analysis of frequencies in multi-way contingency tables. The data used are categorical. Continuous variables may be dichotomized at the median or trichotomized at the 33rd and 66th percentiles for this analysis. This person-centered method looks at patterns or configurations that are basically combinations of characteristics. The key element of configural frequency analysis is that observed frequencies are compared with expected frequencies.

What typical research question can be answered by configural frequency analysis? We would suggest an example from the field of developmental psychology. Imagine that we are investigating a combination of (secure/insecure) parental attachment, (high/low) social skills, and extraversion/introversion with regard to (healthy/abnormal) psychological development in children. While analyzing a dataset, we compare the observed frequencies with the expected frequencies. The expected frequencies could be calculated under the assumption of independence of the relevant psychological constructs. If they were not independent, the observed frequencies would deviate significantly from the expected frequencies, resulting in a pattern or configuration that occurs either more often than expected or less often. If for instance, the observed frequencies occur more often than expected for secure attachment, high social skills and extraversion with regard to healthy psychological development, this could be seen as a common or typical configuration for healthy psychological development. In addition, the configuration of insecure attachment, low social skills, and introversion could be observed significantly less often than expected with regard to healthy psychological development. Therefore, one would infer that this pattern or configuration is uncommon or atypical for a healthy psychological development.

Instead of analyzing means, variances and covariances of scale sores (as in the common variable-centered approach), the personcentered approach analyses units, persons or objects grouped according to their characteristic patterns or configurations in contingency tables (Stemmler \& Lösel, 2012; Stemmler \& von Eye, 2012). Patterns or configurations that occur significantly more often than expected under the null hypothesis are called types. Wickens (1989) used the term 'outlandish cells'. Configurations occurring significantly less often than predicted under the null hypothesis are called antitypes (Krauth \& Lienert, 1973). From a statistical point of view this approach is associated in Germanspeaking countries with the name of Gustav A. Lienert, the Austrian physician and psychologist who invented configural frequency analysis. In the English-speaking world configural frequency analysis is closely associated with the name of Alexander von Eye (von

\footnotetext{
1 Friedrich-Alexander Universität Erlangen-Nürnberg, Germany

2 Technische Universität München, Germany

Corresponding author:

Mark Stemmler, Friedrich-Alexander Universität Erlangen-Nürnberg, Lehrstuhl für Psychologische Diagnostik, Methodenlehre und Rechtspsychologie, Nägelsbachstraße 49c, 91052 Erlangen, Germany. Email: mark.stemmler@fau.de
} 
Eye, 1990, 2002). Von Eye discovered the close relationship between configural frequency analysis and log-linear modeling (LLM) and he is responsible for a number of further statistical improvements to configural frequency analysis, such as parametric configural frequency analysis (Spiel \& von Eye, 1993), autoassociation configural frequency analysis (von Eye, Lerner, Lerner, $\&$ Bowers, 2012) and configural frequency analysis based on Bayes theorem (von Eye \& Gutiérrez-Peña, 2004).

In the following sections, four variants of configural frequency analysis will be presented: (1) first-order configural frequency analysis; (2) prediction configural frequency analysis; (3) two-group configural frequency analysis; and (4) functional configural frequency analysis, which demonstrates the pitfalls of configural frequency analysis using a data example with a cross-table including outlier cells. All variations can be run with the newly developed $\mathrm{R}$-package confreq, which is introduced and explained for hands-on computational analyses.

\section{The Developmental Psychological Background of the Person-centered Approach}

In this article person-centered methods will be presented from a developmental as well as quantitative, statistical approach. In the field of developmental psychology this approach is mainly represented by David Magnusson and Lars Bergman and their research group at the university of Stockholm in Sweden. They describe their approach as holistic and interactionistic, where the individual is seen as an integrated, biological, and social entity. According to von Eye, Bergman, and Hsieh (2015), developmental processes possess three basic characteristics:

1. "The individual organism can be thorougly understood only as a whole, not as a sum of fragmented elements.

2. The unit of person-oriented analysis is indivisible and can, thus, be studied most fruitfully taking a holistic perspective.

3. The key principle of functioning and development of an integrated individual is that of functional interaction." (von Eye, Bergman, \& Hsieh, 2015, p. 796).

The wholeness of an individual evolves out of the dynamics between the structures and elements of the system. The respective structures and processes of the individual encompass psychological constructs, such as behavior, perceptions, goals, and plans of actions, social norms, motives or values, and biological functioning in the brain and physiological system. These constructs obtain their roles and meanings as part of the interaction between the structures of the systems within the whole individual. "A certain element derives its significance, not from its structure, but from its functional role in the system of which it forms a part" (Magnusson \& Mahoney, 2001, p. 5). Development cannot be understood by studying single factors in isolation from other simultaneously operating factors. The individual and the environment influence each other. The individual is seen as an active agent and producer of his or her own development (cf. Lerner \& Busch-Rossnagel, 1981; Silbereisen \& Noack, 2006). Therefore, the human being is seen as a complex dynamic system, which could be understood ideally only under the holistic interactionistic approach (Magnusson \& Allen, 1983).

According to Bergman, von Eye, and Magnusson (2006), such a holistic approach should be used in all studies involving human development, no matter which level of the organized system is in focus, e.g. the level of cells or the level of cognition and/or behavior. They cite the following recent trends in support for their point of view:

1. An increased interest in psychology in biological processes and their interactions with mental, behavioral and social functioning (see Belsky \& Pluess, 2009; Raine, Venables, \& Mednick, 1997;).

2. An increased appreciation of non-linear developing dynamic systems (see McArdle, 2001).

3. An increased interest in longitudinal studies which apply the person-oriented approach to study psychological, biological, and social functioning (see Lösel, Beelmann, Stemmler, \& Jaursch, 2006; Stemmler, Lösel, Beelmann, Jaursch, \& Zenkert, 2005).

\section{The Advantage of the Person-centered Approach in Developmental Psychology}

According to the Stockholm researchers the variable-oriented approach is reductionist. They argue that it strives to identify the most important psychological dimensions that may explain the individual's behavior, based on generally valid laws that may be depicted in a complex model including mutually interacting subsystems. This suggests that the researcher is more interested in sustaining the model than in the developing person. To put it another way: individuals are reduced to delivering score values on measured scales (Magnusson \& Allen, 1983). According to Magnusson and Bergman and their research team, psychological models are based on a system of interrelated variables that cannot be transformed to an individual who is characterized by a complex and specific set of characteristics. In the variable-oriented approach constructs such as aggression, intelligence, or psychopathology are looked at in isolation, but "the reality is the person as a totality, who views the external world from a certain perspective and who functions as an ongoing system" (Magnusson \& Allen, 1983, p. 372). The personcentered approach tries to investigate the developing individual based on its characteristic patterns or structured configurations. Based on homogeneous subgroups of individuals sharing the same patterns of characteristics it is possible to compare different homogenous subgroups and to infer or generalize to a population of similarly structured people. Such a generalization is based on existing individuals and not on existing psychological variables. If the reference is to the human being as a whole, one avoids the fragmentation and the neglect of important self-organizing processes.

The person-centered approach is especially interested in the general principles of the continuous individual-environment interaction processes. The goal is to understand and explain the adaptive processes of maturation and learning of the developing individual. Within the variable-oriented approach the scientific focus is driven by the statistical fit of the proposed prediction models. The individual is in a constant process of change over time. Therefore development should be depicted as a process of constantly changing states (Bergman, Magnusson, \& Khouri, 2003). A state is a specific configuration that determines a system at a certain point in time. The Swedish researchers postulate five tenets regarding the study of the process of change, which are also most important for statistical analysis.

1. Such developmental processes are neither valid for all individuals nor specific for one individual. These processes 
apply always to a subset of individuals and are therefore partly specific.

2. For each developmental process there are always a number of systems involved on different levels which, in addition, influence each other in complex ways.

3. For individual growth there are inter-individual differences based on coherence and structure.

4. The developmental process is based on general principles determined by specific patterns of characteristics and their interacting functional systems.

5. Although it is theoretically possible to come up with an infinite number of possibilities in each subsystem, there is a reduction of those possibilities on the more general level. Therefore, only a few typical configurations evolve as socalled common types. The same is true for intra- and interindividual development.

Bergman and Magnusson (1997) do not exclusively focus on common developmental types, they also look at relatively rare occurring patterns or configurations, so-called antitypes. Antitypes may be based on measurement error, but they also provide the basis for an extension or revision of existing theories.

\section{Introduction to Configural Frequency Analysis}

Configural frequency analysis is the statistical method appropriate to the holistic interactionistic approach of Magnusson. In the following section, we explain the basics of configural frequency analysis according to Lienert and von Eye. The basis is the analysis of frequencies in multi-way contingency tables. In such crosstabulations individuals, animals, or objects are grouped into disjunct categories based on their respective patterns or configurations. The underlying logic is the comparison of observed frequencies $\left(o_{i j k}\right)$ with expected frequencies $\left(e_{i j k}\right)$. There are a number of statistics available for comparing observed with expected frequencies. We will explain the method of configural frequency analysis with a data example of a three-way cross-table, although configural frequency analysis can be applied to any size of a cross-table. Here, we start to calculate a global Chi-square value using equation (1).

$$
\chi^{2}=\sum_{i=1}^{R} \sum_{j=1}^{C} \sum_{k=1}^{S} \frac{\left(o_{i j k}-e_{i j k}\right)^{2}}{e_{i j k}}
$$

$R=$ number of rows ranging from $i=1,2, \ldots, I$

$C=$ number of columns ranging from $j=1,2, \ldots, J$

$S=$ represents the levels of the third dimension, called space, from $k=1,2, \ldots, S$

$o_{i j k}=$ the observed frequencies of row $i$, column $j$ and the third dimension $k$

$e_{i j k}=$ the expected frequencies of row $i$, column $j$ and the third dimension $k$

$o_{. j .}=$ the index-point notation is applied, and the dots denote the observed frequencies of column $j$

$o_{i . .}=$ the dots denote the observed frequencies of row $i$

The associated general formula for the degrees of freedom for any contingency table is defined by equation (2).

$$
d f=T-\sum_{i=1}^{D}\left(v_{d}-1\right)-1
$$

In this equation $T$ representsthe number of cells or configurations, $d=1 \ldots D$ represents the number of variables (dimensions), and $v_{d}$ the number of categories of a variable. Here, we have $T=8$ cells, $D=3$ variables and $v_{d}=2$ categories, that is, $d f=$ $8-(2-1)-(2-1)-(2-1) 1=4$.

An important alternative statistic to the Pearson Chi-square and measure of the goodness-of-fit is the Likelihood Ratio Chi-square (LR); see equation (3).

$$
\mathrm{LR}=2 \sum_{i=1}^{I} \sum_{j=1}^{J} \sum_{k=1}^{K} o_{i j k} \ln \frac{o_{i j k}}{e_{i j k}}
$$

$\ln =$ the natural logarithm for base $e$ (i.e. Euler's constant $=$ $2.7182 \ldots$ )

The total sample size $n$ is $o_{\ldots}=\sum_{i=1}^{I} o_{i . .}=\sum_{j=1}^{J} o_{. j .}=\sum_{k=1}^{K} o_{. . k}$. The global Chi-square tests the following null $\left(H_{0}\right)$ and alternative hypothesis $\left(H_{l}\right)$.

$H_{0}$ : There is no significant discrepancy between the observed and the expected cell frequencies.

$H_{1}$ : There are significant discrepancies between the observed and the expected cell frequencies.

The expected values or frequencies $\left(e_{i j k}\right)$ are defined according to the null hypothesis or a base model (e.g. the assumption of independence). The base model of independence is only one of many possible models to constitute the null hypotheses; different base models result in different null hypotheses and, therefore, in different ways of calculating the expected frequencies. Under the assumption of independence, the expected frequencies are calculated according to the following equations (4) and (5)

$$
\begin{gathered}
e_{i j k}=n p_{i j k} \\
p_{i j k}=p_{i . . p_{. j .} . . k} \quad \text { e.g., } p_{111}=p_{1 . . p_{.1 .} p_{. .1}}
\end{gathered}
$$

$e_{i j k}=$ expected frequencies (first subscript $=$ row; second subscript $=$ column; third subcript $=$ space)

$p_{i j k}=$ cell proportion;

$p_{i .}=$ row proportion;

$p_{. j}=$ column proportion;

$p_{. . k}=$ space proportion.

Expected frequencies and the null hypothesis for a three dimensional cross-table are given by (6) and (7)

$$
\begin{gathered}
H_{0}: \pi_{i j k}=\pi_{i . .} \pi_{. j .} \pi_{. . k} \\
e_{i j k}=\frac{o_{i . . .} . o_{. . k}}{n^{2}}
\end{gathered}
$$

However, a configural frequency analysis can be run on tables of any size. In addition, we differentiate between a local and a global Chi-square value. A local Chi-square compares the observed frequencies with the expected frequencies on the cell level. A global Chi-square value compares the observed with the expected frequencies for the whole table. A local Chi-square is calculated by equation (8)

$$
\chi_{i j k}^{2}=\frac{\left(\mathrm{o}_{i j k}-e_{i j k}\right)^{2}}{e_{i j k}} \text { with } d f=1
$$

Significant local Chi-square values are indicators for the existence of types or antitypes. Another valuable statistic in the search of types or antitypes is the Chi-square approximation to the $z$-test as defined in equations (9) and (10)

$$
z_{\left(\frac{\alpha}{2}\right)}^{2}=\chi_{(\alpha)}^{2}
$$


Table I. Data Example from the Erlangen-Nuremberg University Hospital for Children.

\begin{tabular}{|c|c|c|c|c|c|c|}
\hline \multirow{2}{*}{$\frac{\text { Seizures }}{\text { (Tim }}$} & \multirow{2}{*}{$\begin{array}{c}\text { Birth } \\
\text { Weight } \\
\text { I) }\end{array}$} & \multirow{2}{*}{$\begin{array}{l}\text { Intelligence } \\
\text { (Time 2) }\end{array}$} & \multicolumn{2}{|c|}{ Frequencies } & \multirow[b]{2}{*}{ z-Statistic } & \multirow[b]{2}{*}{ Types } \\
\hline & & & Observed & Expected & & \\
\hline No & $>1000 \mathrm{~g}$ & above average & 27 & 21.19 & 1.26 & \\
\hline No & $>1000 \mathrm{~g}$ & below average & 4 & 6.12 & -0.86 & \\
\hline No & $<1000 \mathrm{~g}$ & above average & 12 & 12.95 & -0.26 & \\
\hline No & $<1000 \mathrm{~g}$ & below average & I & 3.74 & -1.42 & \\
\hline Yes & $>1000 \mathrm{~g}$ & above average & 4 & 6.74 & -1.06 & \\
\hline Yes & $>1000 \mathrm{~g}$ & below average & 1 & 1.95 & -0.68 & \\
\hline Yes & $<1000 \mathrm{~g}$ & above average & 2 & 4.12 & -1.04 & \\
\hline Yes & $<1000 \mathrm{~g}$ & below average & 7 & 1.19 & 5.35 & type \\
\hline
\end{tabular}

Notes. Longitudinal data of prematurely born children with seizure status and birth weight with regard to intelligence in kindergarten age, $n=58$.

$$
\begin{aligned}
z_{\left(\frac{\alpha}{2}\right)}^{2} & =\frac{(o-e)^{2}}{e}= \\
z_{\left(\frac{\alpha}{2}\right)}^{2} & =\frac{(o-n p)^{2}}{n p} \\
z & =\frac{(o-e)}{\sqrt{n p}} .
\end{aligned}
$$

In addition to the Pearson Chi-square statistic and the Chi-square approximation to the $z$-test, several other statistics are available in software packages, such as the binomial test, the approximation of the binomial test using Stirling's formula, and the binomial approximation to the z-test (see Stemmler, 2014; von Eye, 1990). These test statistics differ with regard to their statistical power and their statistical assumptions; no statistical test is superior to the others. However, von Eye (1990) mentions that the Chi-square approximation to the $z$-test shows better characteristics in Monte Carlo simulations than the Chi-square statistic.

Let's have a look at an example of longitudinal data from the Erlangen-Nuremberg University Hospital for Children. The data are from a sample of $n=58$ prematurely born babies. Their medical status was measured shortly after birth (i.e. Time 1). The dependent variable is cognitive development or intelligence as assessed at kindergarten age (i.e. Time 2). The prematurely born babies were divided into children with a birth weight of $1=$ more than 1000 grams or $2=$ less than 1000 grams. Intelligence was categorized as $1=$ average and $2=$ below average. In addition whether the children had $1=$ epileptic seizures or $2=$ no epileptic seizures after they were born was also reorded. Configural frequency analysis compared the observed frequencies with the expected frequencies under the base model of independence and came up with the following results (see Table 1).

It is striking that, although the pattern no seizures, more than 1000 grams, intelligence above average appears most frequently, this is not a type or antitype. Types or antitypes exist where there are significant differences between the observed and expected frequencies. The global Chi-square value is $\chi^{2}=35.43(d f=4$, $p<.05)$ and the related Likelihood-Ratio-value (LR) is $\chi_{L R}^{2}=21.62(d f=4 ; p<.05)$. Fortunately, the outcome was positive for the majority of children $(n=44)$ and their intelligence was above average in kindergarten. However, for a small but significant group of children there was an unfortunate configuration of characteristics: Those children with epileptic seizures and a birth weight below 1000 grams are likely to develop a below average intelligence in kindergarten. The corresponding $z$-value was significant even after the Bonferroni alpha adjustment: $\alpha_{a d j .}=.05 / 8=.00625 ; z=2.497$. Although we are searching for types and antitypes, the standard alpha level is still $\alpha=.05$; for more information, the interested reader should consult von Eye and Gutiérrez-Peña (2004).

Future research should focus on this particular group for early intervention. One feature that turned out to be not relevant in terms of predicting normal intelligence in kindergarten age children was intubation after birth. Intubation showed in our data no negative impact on the cognitive development of children; other nonsignificant results may also reveal interesting information. Caution is necessary with generalizations, however, and findings should be replicated in other independent clinical samples.

A short note on categorizing continuous variables. Configural frequency analysis compares observed frequencies with expected frequencies. It would be ideal if the data at hand existed as natural frequencies, e.g. epileptic seizures (yes or no). In most cases, however, the researcher will be faced with a mixture of categorical and continuous variables. In such a case one may dichotomize the continuous variable at the median or trichotomize the variable at the 33 rd and 66th percentiles. Categorizing quantitative measures is a controversial topic among statisticians because it has serious consequences. The two major consequences are (1) by categorizing a continuous variable one loses important information (about the mean and the variance) and (2) one looses statistical power. Therefore, such a practice should not be done without thorough consideration. An article often cited by the opponents of categorization is MacCallum, Zhang, Preacher, and Rucker (2002). They argue against categorizing continuous variables, because there is always a certain arbitrariness involved (e.g. why is $1000 \mathrm{~g}$ a meaningful cutoff for prematurely born babies?) and participants close to but on different sides of the cut-off are treated as very different, although they might share great similarity. However, MacCallum and colleagues mainly criticize categorizing quantitative measures that are then used in ordinary multivariate analysis such as multiple regression or ANOVA. There is no mention in their article of the use of categorical variables in LLM.

The configural frequency analysis presented above is based on the assumption of independent main effects and is an example of first-order configural frequency analysis. The developer of configural frequency analysis, Lienert, wanted to use configural frequency analysis only for exploratory purposes (Stemmler, Lautsch, \& Martinke, 2008), but with Bonferroni alpha adjustment it is possible to test hypotheses (Lehmacher, 2000). In addition to Bonferroni adjustment, there are at least two other useful procedures for the protection of the $\alpha$-level. One is by Holland and Copenhaver (1987) and the other is by Hommel (1988). For the analysis of patterned frequencies via configural frequency analysis the R-package confreq (Heine, 2015) is available for the statistical software environment $R$ ( $R$ Development Core Team, 2015). $\mathrm{R}$ is an open source statistical software suitable for most operating systems such as Linux, MacOS X and Windows.

\section{Conducting a First-order Configural Frequency Analysis Using the R-package confreq}

The results presented above can be replicated easily by using the R-package confreq (Heine, 2015), for the freely available statistical 


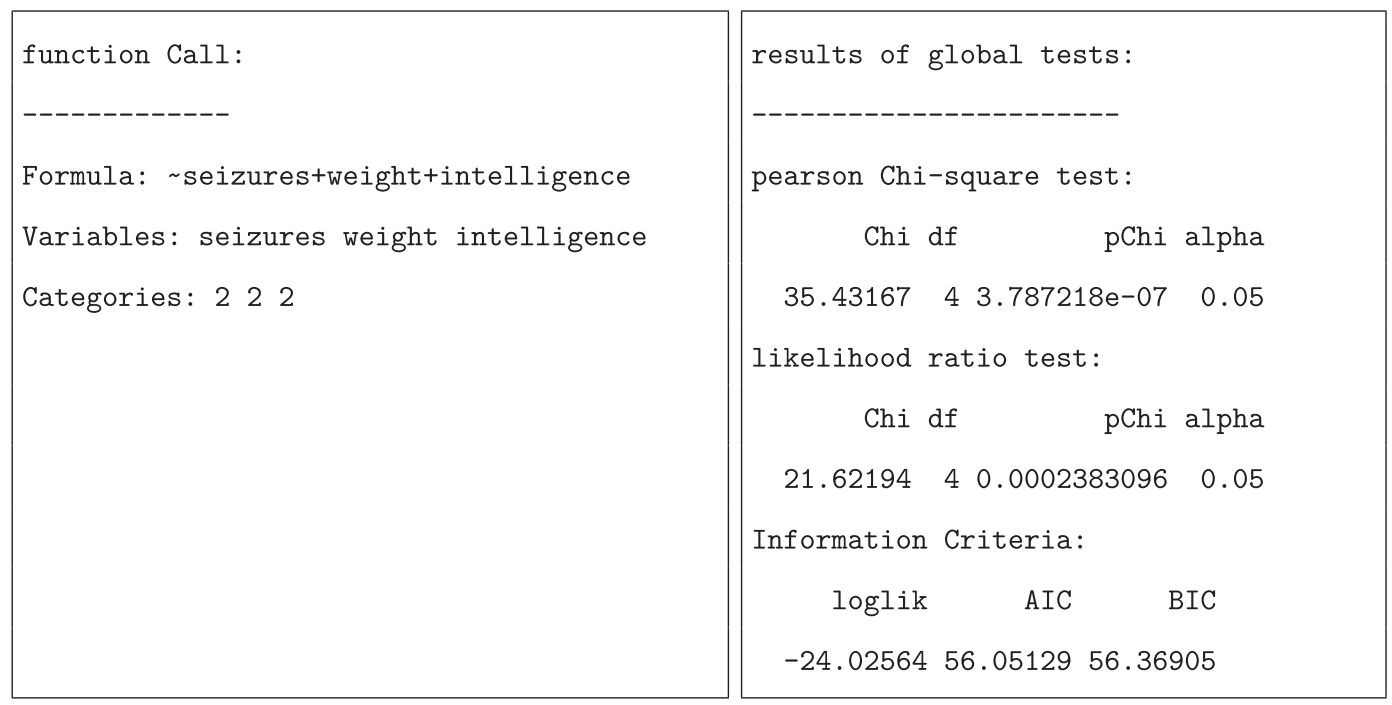

Figure I. R-console Output for Function Call (Left Display) and Results of the Global Test (Right Display) as Returned by the Function CFA ( ) for the Erlangen-Nuremberg University Hospital Data.

software R (R Development Core Team, 2015). R and the package confreq are both available from the Comprehensive $\mathrm{R}$ Archive Network website (CRAN) at http://cran.r-project.org/index.html. The core principle in the package confreq is to use LLM using the function $g \operatorname{lm}()$ within the stats base package to compute the expected counts, based on a model (design) matrix. The main functions are CFA() and S2CFA() (see package description Heine, 2015). In the following section it is assumed that the user has already set up a basic $\mathrm{R}$ environment on the computer and has already made some elementary experience in dealing with $\mathrm{R}$. The complete beginner should consult the introductory literature on $\mathrm{R}$ (see, for example, Alexandrowicz, 2013).

To replicate the example given in the above section (see Table 1) we first have to setup the data in a tabulated form for the analysis. Therefore start $\mathrm{R}-$ or your preferred R-gui-editor and type in and execute the command lines 1 to 5 given in listing 1 .

Listing $1 . \mathrm{R}$ commands to generate the example data.

1. seizures <- c('no", "no", "no', "no", 'yes', 'yes", 'yes', 'yes")

2. weight <- c(">1000gr", ">1000gr", "<1000gr", "<1000gr", ">1000gr", ">1000gr", "<1000gr", "<1000gr")

3. intelligence <- c( "above average", "below average", 'above average", 'below average', 'above average", "below average", "above average", "below average")

4. Freq. $\mathrm{d} 1<-\mathrm{c}(27,4,12,1,4,1,2,7)$

5. $\mathrm{d} 1<-$ data.frame (seizures, weight, intelligence, Freq $=$ Freq $\cdot$ d1)

The commands will produce an R-object named ' $\mathrm{d} 1$ ' containing the configurations for the three dichotomous variables and their respective frequencies as already depicted in Table 1.

To proceed with the configural frequency analysis using the package confreq the R-object ' $\mathrm{d} 1$ ' must be assigned a special class. Thus, type in and execute the command line number 1 given in listing 2 into the R-console.
Listing 2. $\mathrm{R}$ commands to prepare the data, compute and show the results of configural frequency analysis.

1. class $(\mathrm{d} 1)<-\mathbf{c}$ ("data.frame", "Pfreq")

2. resd1<- CFA(d1, form = " seizures+weight+ intelligence")

3. $\quad$ summary (resd1, sorton = "pat. ", showall = TRUE)

To perform the final analysis, type in the command line number 2 given in the listing 2 . This will assign the results from applying the function $\mathrm{CFA}$ ( ) to the data in ' $\mathrm{d} 1$ ' to an object named 'resd1'.

By defining the argument form = "seizures+weight+ intelligence" the function $\mathrm{CFA}$ () will calculate the results for a configural frequency analysis based on the configurations and observed frequencies given an object ' $\mathrm{d} 1$ ' considering only the main effects of the three variables. In order to output the results on the R-console the summary() function for the result object 'resd1' can be used, this entails executing the command line number 3 given in listing 2 .

In addition to the results in Table 1 some supplementary information and coefficients are displayed. First, the formula definition, which was used to compute the expected frequencies and the variables in the tabulated data with their respective number of categories, are returned. Second, the results of the global tests of significance ( $\chi^{2}$ and likelihood ratio test) are displayed on the screen. Furthermore, the likelihood and the information criteria AIC and BIC for the respective model are given. (see Figure 1).

Referring to Table 1, the results of the local tests received through the summary () function are depicted below in Figure 2.

The first column of the output named 'pat.', shows all possible patterns or rather all configurations for the three dichotomous variables. The next column holds the observed frequencies of the respective configuration. In the third column named 'exp.' the expected frequencies, calculated according to the model definition as given in the argument form of the function $\mathrm{CFA}$ ( ), are shown. Columns five to seven contain the results of the local Chi-square tests.

To render the output easily comprehensible, a symbol is used in column four ('Type'), indicating whether the respective configuration is either a type (+), antitype (-) or not significant at all (.). By 
results of local tests:

results of local tests returned by the function CFA()

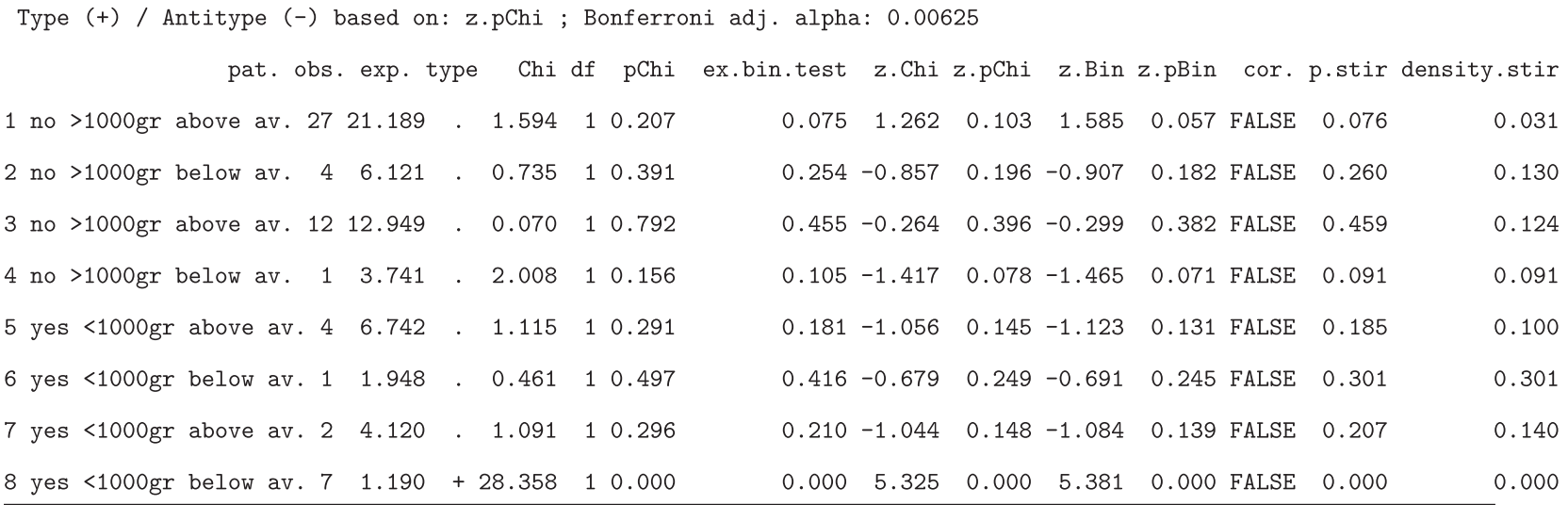

Figure 2. R-console Output of the Local Test as Returned by the Function CFA ( ) for the Erlangen-Nuremberg University Hospital Data.

default this decision is based on the comparison of the Bonferroni adjusted alpha with the $p$-value of the Chi-square approximation to the $z$-test (' $z$.pChi' - see column 10 in Figure 2).

Columns eight to 15, in Figure 2, contain the results of several additional local tests. These are (from left to right) the $p$-value of the exact binomial test ('ex.bin.test'), the Chi-square approximation to the $z$-test (' $z$.Chi') with its corresponding $p$-value (' $z$.pChi') and the binomial approximation to the $z$-test (' $z$. Bin') with its corresponding $p$-value ('z.pBin'). The column with the subscript 'cor.' displays information on whether a continuity correction was applied when calculating the two approximation tests. Finally the last two columns hold the results of the binomial approximation using Stirling's formula (e.g. Stemmler, 2014, p.20).

\section{Configural Frequency Analysis and LLM}

This section describes the close relationship between configural frequency analysis and LLM. LLM is a well-used method for the analysis of multiway contingency tables (e.g. Agresti, 2013; von Eye \& Mun, 2013). LLM is used to identify the structure among the categorical variables under investigation. It parametrizes the distribution of cell frequencies, or to put it another way, the logarithms of cell frequencies, in terms of main effects and interactions. For a two-by-two cross-table the expected frequencies are parametrized as follows in equation (11) for the saturated model

$$
\ln e_{i j}=\lambda_{0}+\lambda_{i} A_{i}+\lambda_{j} B_{j}+\lambda_{i j} A_{i} B_{j}
$$

$\ln =$ the natural logarithm for base $e$.

Equation (11) represents the log-linear model, which estimates the $e_{i j}$ as a linear combination consisting of a constant, two main effects and an interaction effect. The $\lambda \mathrm{s}$ are parameters weights of the variables or variable interactions of the expected frequencies. They are not observed from the data but estimated and they can be interpreted as regression effects or dummy-weights in multiple regression. Equation (11) also reveals the linear as well as additive characters of LLM. Configural frequency analysis and methods of LLM are descendants of the generalized linear model (GLM) (Agresti, 2013). The equation of the GLM is as follows (12)

$$
f(Y)=X \beta
$$

where $Y$ is the dependent variable, $X$ is the matrix of independent variables, and $\beta$ is the parameter vector, which contains the weights with which the independent variables explain the dependent variable. The function $f(Y)$ is called the link function and describes the transformation performed on the dependent variable. For the loglinear model, the link function is a logarithmic function (i.e. $\log (E)$ ), called the log-link (cf. von Eye, 2002). The log-link is specified as given in (13).

$$
\log (E)=X \lambda
$$

For $\log (E)$ we use the natural logarithm (14)

$$
\ln e_{i j k}=m_{i j k} \text {. }
$$

$\ln e_{i j k}$ is the logarithm of the expected frequencies. The general linear model is a special case of the GLM and the following formulas reveal the close relationship between the two models. In the general linear model $\beta$ is calculated by (15)

$$
\left.\beta=\left(X^{T} X\right)^{-1} X^{T}\right) Y
$$

and if we replace $\beta$ with $\lambda$ the parameters are given by (16)

$$
\left.\lambda=\left(X^{T} X\right)^{-1} X^{T}\right) \log (m)
$$

$X$ is the design matrix containing the effect-coded main effect and interaction terms plus the constant. The design matrix $X$ has as many rows as there are cells or configurations, and $w+1$ columns. $w$ is the number of weights; the first weight is always the constant, coded with ones. $m$ is the vector of the logarithms of the expected cell frequencies.

Let's look again at our example in Table 1. The goal of LLM is to investigate the underlying structure of the data with the help of main effects or interactions. Therefore, we start with a model, assuming independence between the variables. This is the so-called main-effects model. Significant main effects indicate that the row and column marginal are not uniformly distributed. The expected frequencies are estimated according to equation (17) and under the null hypothesis of independence among the three variables $(A, B$ and $C$ ) we obtain the expected frequencies as for example listed in Table 1

$$
\ln e_{i j}=\lambda_{0}+\lambda_{i} A_{i}+\lambda_{j} B_{j}+\lambda_{k} C_{k}
$$


The expected frequencies have two characteristics:

1. the marginal values for the expected frequencies of variable $A, B$ and $C$ are equal to the marginal values of the observed frequencies, and

2. the total sample size is reproduced and it holds $\sum_{i=1}^{I} \sum_{j=1}^{J} \sum_{K=1}^{k} e_{i j k}=o_{. .}=n$

If one compares the above observed with the expected values, one might detect large differences between the observed and expected frequencies. Therefore, our underlying main-effects model may not hold. What does the corresponding design matrix for the above data look like? The main effects and interaction terms are effect coded; that is, we use coefficients $c_{i}$ for each category of a variable, which have to sum to zero.

We have two categories for variable $A$ (Seizures), and we used the coefficients 1 and -1 . We do the same with variable $B$ (Birth Weight) and variable $C$ (Intelligence). The design matrix is below:

$$
X=\left(\begin{array}{rrrr}
1 & 1 & 1 & 1 \\
1 & 1 & 1 & -1 \\
1 & 1 & -1 & 1 \\
1 & 1 & -1 & -1 \\
1 & -1 & 1 & 1 \\
1 & -1 & 1 & -1 \\
1 & -1 & -1 & 1 \\
1 & -1 & -1 & -1
\end{array}\right)
$$

The values (1) in the first column represent the constant, the other columns display the coefficients for the main effects of variable $A, B$, and $C$. The assumption that the observed frequencies are reproduced from the marginal values means that these variables have a certain effect on the expected frequencies. For LLM it is important to know how big these effects are (in terms of the regression weights). These weights can be found in the following equation (19) for the saturated model

$$
\begin{aligned}
e_{i j k}= & \gamma_{0} \times \gamma_{i} A_{i} \times \gamma_{j} B_{j} \times \gamma_{k} C_{k} \times \gamma_{i j} A_{i} B_{j} \times \gamma_{i k} A_{i} C_{k} \times \gamma_{j k} B_{j} C_{k} \\
& \times \gamma_{i j k} A_{i} B_{j} C_{k}
\end{aligned}
$$

This can be transformed into an additive relationship via the natural logarithm (20).

$$
\begin{aligned}
\ln e_{i j k}= & \lambda_{0}+\lambda_{i} A_{i}+\lambda_{j} B_{j}+\lambda_{k} C_{k}+\lambda_{i j} A_{i} B_{j} \times+\lambda_{i k} A_{i} C_{k} \\
& +\lambda_{j k} B_{j} C_{k}+\lambda_{i j k} A_{i} B_{j} C_{k}
\end{aligned}
$$

Equation (20) represents the saturated log-linear model, which estimates $e_{i j k}$ as a linear combination consisting of a constant, three main effects, three first-order interactions, and one second-order (triple) interaction.

Data in Table 1 are entered in $\mathrm{R}$ as pattern frequencies, where the first number represents the first categorical value of the first variable (row variable), the second number the first categorical value of the second variable (column variable), the third variable (third dimension) comes next, at the very end the frequencies are listed. The rule of thumb is: The last variable cycles first! To assess how much the expected frequencies deviate from the observed frequencies R-package confreq accommodates two goodness-offit-statistics: (1) the Pearson Chi-square and the (2) Likelihood Ratio Chi-square (LR).

A model fits or the observed frequencies do not deviate significantly from the expected frequencies if the respective Chi-square or LR-values has a $p$-value greater (!) than $\alpha=.05$. Here, both $p$-values of the main effects model indicate a lack of fit: $\chi_{L R}^{2}=21.62194, d f=4, p<.05 ; \chi^{2}=35.43167, d f=4$, $p<.05$. To examine the importance of the interaction term, a saturated model is calculated. A saturated model may appear tautological, because the expected frequencies reproduce exactly the observed frequencies and the respective Pearson-Chi-square and LR-statistic are always zero. But for didactic reasons it is presented here (please note, that base models for configural frequency analysis are never saturated and log-linear parameters are rarely interpreted in configural frequency analysis). In a saturated model all lambda parameters are estimated. Let's look at the design matrix of the saturated model (21)

$$
X=\left(\begin{array}{rrrrrrrr}
1 & 1 & 1 & 1 & 1 & 1 & 1 & 1 \\
1 & 1 & 1 & -1 & 1 & -1 & -1 & -1 \\
1 & 1 & -1 & 1 & -1 & 1 & -1 & -1 \\
1 & 1 & -1 & -1 & -1 & -1 & 1 & 1 \\
1 & -1 & 1 & 1 & -1 & -1 & 1 & -1 \\
1 & -1 & 1 & -1 & -1 & 1 & -1 & 1 \\
1 & -1 & -1 & 1 & 1 & -1 & -1 & 1 \\
1 & -1 & -1 & -1 & 1 & 1 & 1 & -1
\end{array}\right)
$$

The interaction terms are the result of the multiplication of the three main effects, therefore we have four interaction terms $A \times B$, $A \times C, B \times C$, and $A \times B \times C$ (see the number of columns is equal to the number of lambdas in equation (20).

By looking at the $z$-standardized lambda parameters, one can see that only intelligence is a significant main effect: seizures $\left(\lambda_{A}=1.74265\right)$, intelligence $\left(\lambda_{B}=2.50737\right)$, and for weight $\left(\lambda_{C}=0.52370\right)$. The interaction term seizures $\times$ intelligence is also significant $\left(\lambda_{A \times B}=2.36331\right)$; the others are not: seizures $\times$ weight $\left(\lambda_{A \times C}=1.90900\right)$, intelligence $\times$ weight $\left(\lambda_{B \times C}=1.14437\right)$, and seizures $\times$ intelligence $\times$ weight $\left(\lambda_{A \times B \times C}=-1.77595\right)$. In our case, a model that reproduces the observed frequencies satisfactorily needs to include the interaction terms seizures $\times$ intelligence and seizures $\times$ weight; no better or more parsimonious model is available. In addition one can see that the observed values are perfectly reproduced, which is constitutive for the saturated model.

\section{Prediction Configural Frequency Analysis}

Prediction configural frequency analysis and the two-group configural frequency analysis use the same base model. Prediction configural frequency analysis defines independent and dependent variables, especially in longitudinal research.See the online appendix for a special example of prediction configural frequency analysis, its specific research questions, and the R-syntax.

\section{Two-group Configural Frequency Analysis}

In the following, two-group configural frequency analysis (Stemmler \& Bingham, 2004; Stemmler \& Hammond, 1997; von Eye, 2002; von Eye, Mair, \& Eun-Young, 2010 ) is illustrated. The underlying assumption representing the null hypothesis is that two groups or samples are drawn from the same population. In other words, let's say $A$ and $B$ are variables characterizing the grouping variable $C$, then the contingency tables of $A$ and $B$ together need to be homogenous across $C$. The underlying null hypothesis is $H_{0}: \pi_{A B C}=\pi_{A B} \pi_{C}$ with the respective alternative hypothesis $H_{1}: \pi_{A B C} \neq \pi_{A B} \pi_{C}$. 
Table 2. Data Example for Two-group Configural Frequency Analysis from Stemmler and Masendorf (1998).

\begin{tabular}{|c|c|c|c|c|c|c|c|}
\hline \multicolumn{2}{|c|}{ Change in Ability } & \multicolumn{2}{|c|}{ Group } & \multirow{2}{*}{$\begin{array}{l}\text { Expected } \\
\text { Frequencies }\end{array}$} & \multirow[b]{2}{*}{$p^{\prime}$} & \multirow[b]{2}{*}{$\chi^{2}$} & \multirow[b]{2}{*}{ Types } \\
\hline Reasoning & Mathematical & Control & Experimental & & & & \\
\hline+ & + & 3 & 4 & 3.5 & 0.291 & 0.166 & \\
\hline+ & - & 4 & 4 & 4.0 & 0.298 & 0.000 & \\
\hline- & + & 4 & 15 & 9.5 & 0.001 & 10.272 & $\mathrm{~T}$ \\
\hline- & - & 14 & 2 & 8.0 & 0.000 & 13.235 & $\mathrm{~T}$ \\
\hline
\end{tabular}

Notes. Observed and expected frequencies for two groups, $n=50$ ( 25 per group) in a two-group configural frequency analysis; Coding: '-' is incremental change below median and ' + ' is incremental change above median; ' $P$ value of Fisher's exact test.

The following (slightly modified) data example is taken from Stemmler and Masendorf (1998). It is an evaluation of a combined training group compared to a control group. The intervention consists of a training called EURO-Mulli (Angerheofer, Kullik, \& Masendorf, 1992) and a training focused on enhancing problem solving and reasoning (Klauer, 1989). Both trainings strengthen students' ability in problem solving and reasoning. The experimental and the control group each consisted of 25 students from a school for learning disabled children in the city of Rostock, Germany. The control group received regular schooling, the experimental group received the training in addition to regular schooling. The dependent variables were measures of maths ability and a test for reasoning ability.

There were two assessments, before and after the training. The patterns were coded as ' + ' if the incremental change was above the median of the ordinary growth or change of the knowledge of the students, and '-' if the incremental change was below the median of the ordinary growth or change of the knowledge of the students. As one can see from Table 2 the expected frequencies are the same for the two groups with regard to each pattern. This fact is data-specific, not method-specific. If there were different sample sizes, the expected frequencies would have been different too. Two statistics are available, the Pearson Chi-square values and the Fisher's exact text. Fisher's test is exact; no assumptions need to be made concerning an approximation of a test statistic to a sampling distribution. Therefore the exact test is preferred over the Chisquare statistic. The resulting $p$-value indicates the probability of the combination of observed frequencies for the pattern under investigation under the null hypothesis of independence. Twogroup configural frequency analysis does not differentiate between types and antitypes; instead discrimination types are identified, which discriminate significantly between the two groups within the sample under investigation. The results suggest that the experimental group profited in terms of their maths ability as a reult of the training. With regard to the pattern ' -+ ' representing a below median growth for reasoning over time but a above median growth for maths ability, there were only four students of the control group but 15 students from the experimental group. Another discrimination type evolved (i.e. '- -') suggesting that more students from the control group than from the experimental group showed a growth below the median in terms of reasoning and maths ability.

\section{Conducting a two-group configural frequency analysis with the R-package confreq}

In this section a new function - S2CFA() - of the R-package confreq is introduced and an alternative method of importing data into $\mathrm{R}$ from a spreadsheet program (such as Excel or Open Office) is demonstrated. To set up your data, open your preferred

\begin{tabular}{|c|c|c|c|c|}
\hline-1 & A & B & C & D \\
\hline 1 & reason & math & group & Freq \\
\hline 2 & + & + & C & 3 \\
\hline 3 & + & - & C & 4 \\
\hline 4 & - & + & C & 4 \\
\hline 5 & - & - & C & 14 \\
\hline 6 & + & + & $E$ & 4 \\
\hline 7 & + & - & $E$ & 4 \\
\hline 8 & - & + & $E$ & 15 \\
\hline 9 & - & $=$ & $\mathrm{E}$ & 2 \\
\hline 10 & & & & \\
\hline
\end{tabular}

Figure 3. Tabulated data $(n=50)$ for a Two-group Configural Frequency Analysis Entered into a Spreadsheet.

spreadsheet program and type in the first row of the first three columns (A, B, and C) the names of the variables: 'reason', 'math,' and 'group'. In the last column (D) enter 'Freq' (this column will hold the observed frequencies of the pattern or configurations defined in columns $\mathrm{A}$ to $\mathrm{C}$ ). Now enter the configurations in the columns A to C. For column A enter in the rows 2 to 9 the following (column vector): ' ++--++-- '. In order to do so, for the most spreadsheet programs you tell the program that you want to enter the + or the - as a character (and not as symbol for a mathematical operation). Therefore, you add an apostrophe before typing the + or the - (e.g. type ' and + ) to get a proper result. For column B enter in the rows 2 to 9 the column vector: ' +-+-+-+- ' and in column C: 'C C C C E E E E' for Control and Experimental group respectively. In the last column (D) named 'Freq' enter the respective frequencies that are ' $3,4,4,14,4,4,15,2$ ' for the data example in the previous section. The final data entry in tabulated form should look like the one depicted in Figure 3.

To prepare the data to be imported into $\mathrm{R}$, save the spreadsheet as an csv file into your current $\mathrm{R}$ workspace directory by naming it 'twogroup.csv'. Depending on the language settings of your operating system or your spreadsheet-program, the field separator for the spreadsheet cells will either be a semicolon or a comma in the csv file. Either format (semicolon or a comma) will be fine for this example but remember the respective format for a proper import of the file into R. After saving the data file, switch to your R editor and type in and execute either of the two commands in the lines 1 and 2 shown in Listing 3. Execute only the line number 1 assuming that the field separator is a semicolon, or the line number 2 assuming that the field separator is a comma.

Once the data is successfully imported, you must define the class of the R-object ' $d 3$ ' to proceed with the two-group configural frequency analysis analysis. Thus, type in and execute the command line number 3 in Listing 3. 


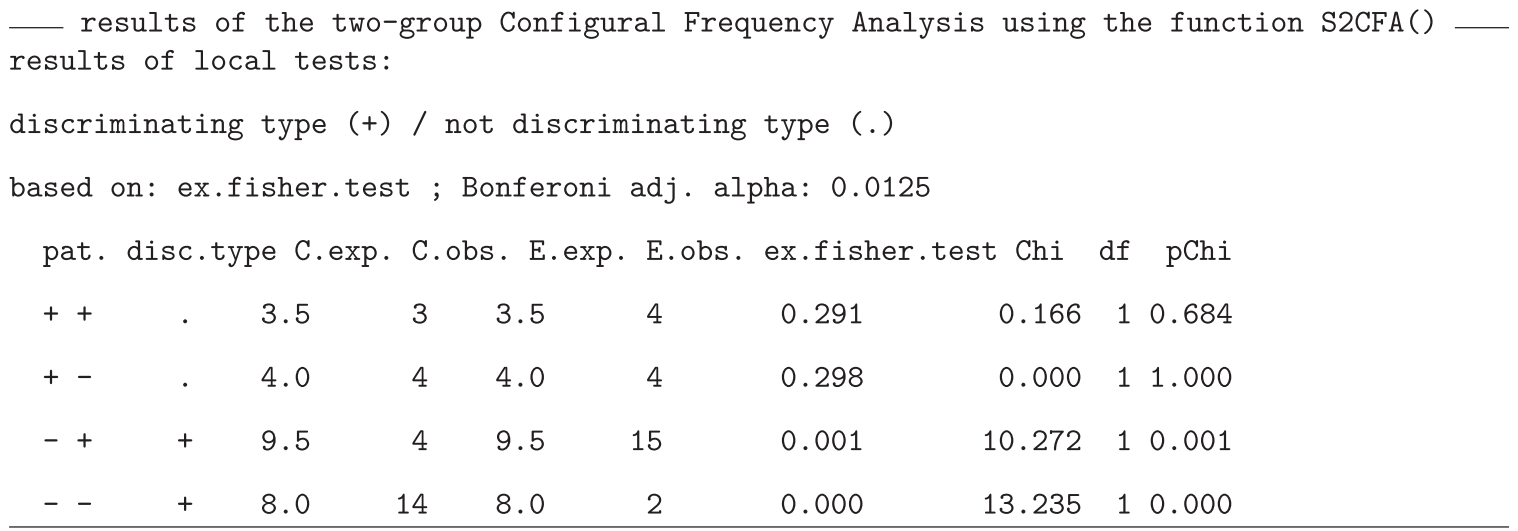

Figure 4. R-console Output of the Results for a Two-group Configural Frequency Analysis for the Stemmler and Masendorf (1998) Data.

Listing 3. R commands to import and prepare the data from a . csv file.

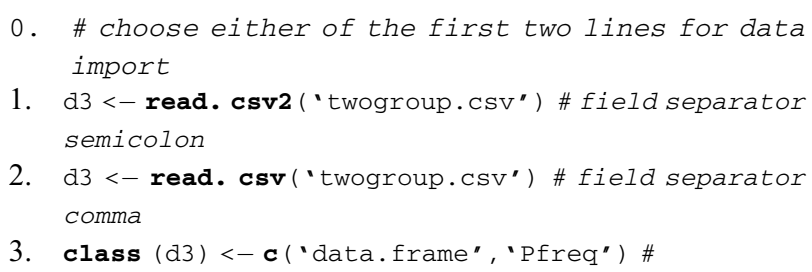

To performthe two-group configural frequency analysis and print the results of the analyses on the screen, execute the two command lines depicted in Listing 4.

Listing 4. R commands to compute and print the results for a two-group configural frequency analysis.

1. resd3 $<-$ S2CFA (d3)

2. summary (resd3)

The summary of the results will look like the one depicted in Figure 4. The first column shows the configurations of the variables under analysis. Columns three to six contain the observed expected cell frequencies for each group (here ' $C$ ' for control group and ' $E$ ' for experimental group). In column seven (ex.fisher.test) the $p$-value of the exact Fisher test is displayed and the last three columns contain the coefficients of the $\chi^{2}$-test. In order to recognize easily which configuration discriminates between the two groups, there is a symbol in the second column indicating whether the respective configuration is either a discrimination type $(+)$ or not (.) based on the $p$-value of the exact Fisher test.

Note that for any analyses using the function $\mathrm{S} 2 \mathrm{CFA}$ ( ) the data in tabulated form must be prepared so that the last column holds the dichotomous (factor) variable defining the two groups. As seen above, two-group configural frequency analysis is a powerful tool for evaluation research!

\section{Functional configural frequency analysis and limits of configural frequency analysis}

The calculation of the expected frequencies depends on the chosen base model. There are in fact at least four different possible models for such a base model or the respective null hypothesis. It is necessary to choose a specific base model with regard to the meaning and content of the base model under investigation. Different base model
Table 3. Three by Three Contingency Table with Two Extreme Outliers.

\begin{tabular}{ccccc}
\hline & $A_{1}$ & $A_{2}$ & $A_{3}$ & $\sum$ \\
\hline$B_{1}$ & 1 & 10 & 10 & 21 \\
$B_{2}$ & 10 & 10 & 10 & 30 \\
$B_{3}$ & 10 & 10 & 370 & 390 \\
\hline$\sum$ & 21 & 30 & 390 & $n=441$ \\
\hline
\end{tabular}

Notes. Example taken from Kieser \& Victor, 1999, p.969.

test different null hypotheses. The first model is the model of independence or a main effects model, which we used above for our firstorder configural frequency analysis. The expected frequencies can be calculated with the help of a log-linear model. This model makes an important assumption; it assumes that each case in the table was drawn from the same population. The second approach, the Victorapproach to configural frequency analysis, was introduced by Victor (1989). With this approach the underlying null hypothesis is based on the assumption, that the configural frequency analysis types or antitypes were drawn from a different population. The third approach is the functional approach to configural frequency analysis (von Eye \& Mair, 2001). The fourth approach was introduced by Gutiérrez-Peña $\&$ von Eye (2000) and Gutiérrez-Peña (2012). It calculates the expected frequencies according to Bayes Theorem. Functional configural frequency analysis and the Victor approach are similar. Both approaches can be applied using LLM software. The functional configural frequency analysis is presented here, because it can be easily run with the R-package confreq.

The limits of configural frequency analysis were first noted in the 1970s (Langeheine, 1980; Wermuth, 1973). Much attention was given to Victor and his colleague Kieser (Kieser \& Victor, 1991, 1999; Victor, 1983). An interesting data example was introduced by Kieser (Kieser \& Victor, 1999, p. 969; see Table 3 below).

From eyeballing Table 3 one would assume one type and one antitype, an antitype in cell or pattern ' 11 ' and a type in ' 33 '. Note, each cell has two indices, ' 11 ' stands for first row and first column with the observed frequency $f_{\text {obs. }}=1$. However, running a firstorder configural frequency analysis brings a surprising result: Cells ' 11 ', ' 13 ', ' 31 ' and ' 33 ' are the only cells that fulfill the condition of independence, all other cells are declared to represent types or antitypes (see R-output in Figure 5). Obviously, the base model of independence was not sensitive enough to detect the type in configuration ' 33 '. The problem is that the assumption of independence is applied to the entire contingency table. To actually 


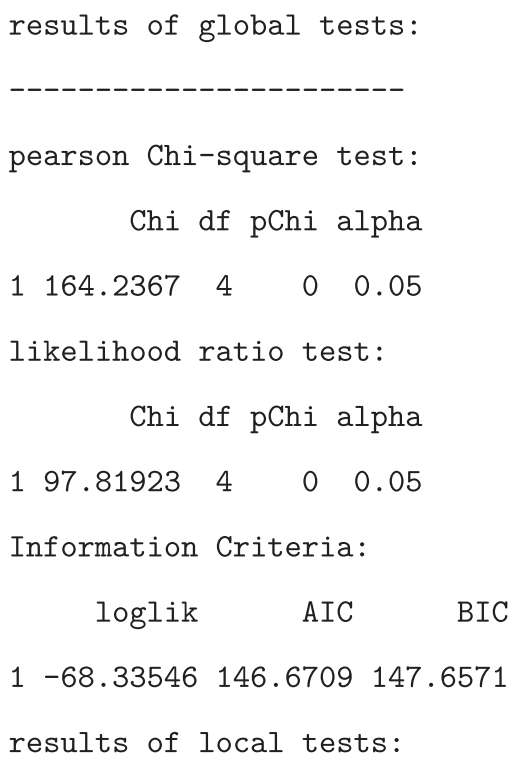

Figure 5. R-console Output of the Results for an Initial First-order Configural Frequency Analysis for the Data from Kieser and Victor (1999), p.969.

detect the obvious type statistically, Victor (1989) suggested treating the outlier cell with pattern ' 33 ' as a structural zero. Structural zeros are usually cells which cannot be observed (e.g. a pattern of heavy rain together with a beautiful blue sky). After confirming the existence of a Victor-type, one has to test whether the rest of the table is independent. If the rest of the table is independent, this is called quasi-independence in the presence of one type. One useful procedure to detect so-called Victor-types is the application of the functional configural frequency analysis (von Eye \& Mair, 2001). Victor-configural frequency analysis and functional configural frequency analysis share the characteristic of blanking out cells. They differ, however, in statistical characteristics and, therefore, do not always lead to the same result. Functional configural frequency analysis uses the design matrix in order to blank out extreme cells, while setting them to a structural zero. Let's take the data from Table 3 and blank out the cell ' 33 ' with the following design matrix

$$
X=\left(\begin{array}{rrrrrr}
1 & 1 & 0 & 1 & 0 & 0 \\
1 & 1 & 0 & 0 & 1 & 0 \\
1 & 1 & 0 & -1 & -1 & 0 \\
1 & 0 & 1 & 1 & 0 & 0 \\
1 & 0 & 1 & 0 & 1 & 0 \\
1 & 0 & 1 & -1 & -1 & 0 \\
1 & -1 & -1 & 1 & 0 & 0 \\
1 & -1 & -1 & 0 & 1 & 0 \\
1 & -1 & -1 & -1 & -1 & 1
\end{array}\right)
$$

The first five columns represent the ordinary main effects model with the constant and the effect-coded main effects. The sixth column blanks out cell ' 33 '. The configural frequency analysis or loglinear model should now result in a fit with no significant residuals left. The elimination of cells can be based simply on searching for the largest residual. The overall goal is to extend the design matrix 


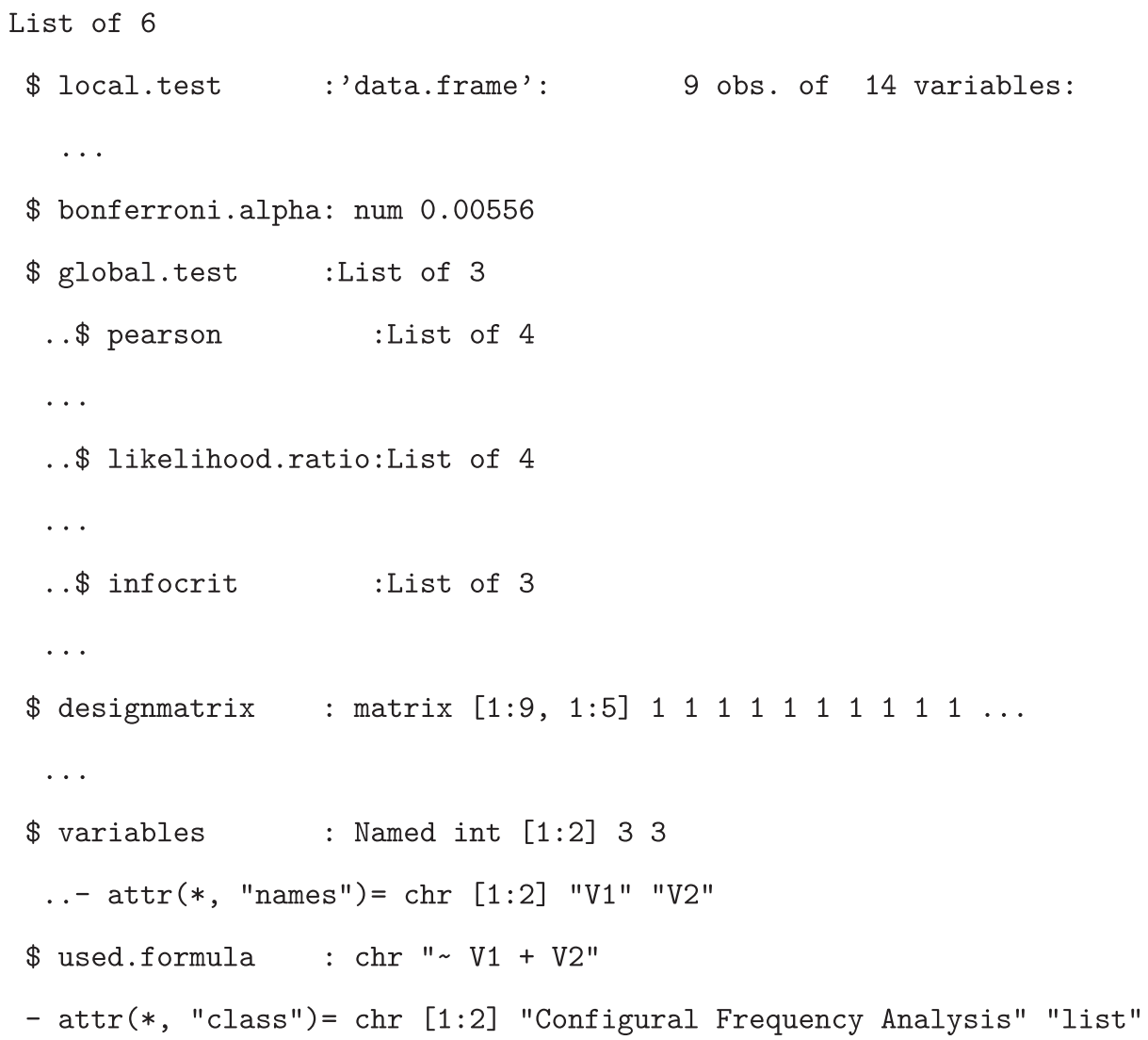

Figure 6. R-console Output of the Results for a Functional Configural Frequency Analysis for the data from Kieser and Victor (1999), p.969.

until the model fits. While first-order configural frequency analysis is a one-step procedure, functional configural frequency analysis is a multiple-step procedure that needs an alpha adjustment. Fortunately, the R-package confreq is able to insert any design matrix in order to fit the structure of the contingency table.

To demonstrate the functional approach of configural frequency analysis using the R-package confreq, we take data from Kieser and Victor $(1999$, p. 969) as mentioned above. Initially we run a firstorder configural frequency analysis on the data. We then use the design matrix for the first-order configural frequency analysis extended in order to eliminate the outlier cell with configuration ' 33 '. In accordance with the examples above in the previous sections, we first prepare the data object in $\mathrm{R}$ by executing the syntax given in listing 5. Command line number 1 in Listing 5 starts with assigning a matrix representing all possible configurations to an object named 'Configs'. The respective frequencies are assigned to 'Freq_d4' in line number 2 and finally a proper class definition is assigned to the final object ' $\mathrm{d} 4$ '.

Listing 5. R commands to set up the data for the functional configural frequency analysis example.

1. Configs $<-$ pos_cfg_cfa $($ kat $=\mathbf{c}(3,3)$, fact $=\mathrm{T})$ \# configurations

2. Freq_d4 <- $\mathbf{c}(1,10,10,10,10,10,10,10,370)$ \# frequencies

3. d4 <-data.frame (Configs, Freq = Freq_d4) \# built tabulated data

4. class (d4)<-- c("data.frame","Pfreq") \# define the "class "

5. a4 \# display tabulated data on screen
To proceed with the initial first-order configural frequency analysis we type in the following command lines which will assign the result to an R-object named 'resd4' and display it on the screen (see Listing 6).

Listing 6. $\mathrm{R}$ commands to compute and print the results for a initial first-order configural frequency analysis.

1. $\operatorname{resd} 4<-\mathrm{CFA}(\mathrm{d} 4$, form $=$ ' $\sim \mathrm{V} 1+\mathrm{V} 2$ ')

2. \# CFA with d4 (main effects model)

3. summary (resd4, showall = TRUE)

The results depicted in Figure 5 show some unexpected significant configurations which obviously result from of the extreme outlier cell ' 33 '.

Besides using the summary ( ) function to present the results on the screen, we can take a closer look at the structure of the resulting object by typing the command str (resd4) into the R-console. This command will reveal the structure of the so-called 'list' object where the results of the former calculations are stored - this is shown in a somewhat abbreviated form in Figure 6.

As we can see, the 'list' object has six major entries named 'local.test', 'bonferroni.alpha', 'global.test', 'designmatrix', 'variables' and 'used.formula' with the entry ' $g$ lobal . test' being a ' 1 ist' itself, containing three (sub)entries named 'pearson', 'likelihood.ratio' and 'infocrit' each of them being also a ' 1 ist'.

Before we proceed with the next step, we shall look at the design matrix underlying the calculations of the first-order configural frequency analysis by typing the command resd 4 designmatrix 


$\begin{array}{rrrrrr} & \text { (Intercept) } & \text { V11 } & \text { V12 } & \text { V21 } & \text { V22 } \\ 1 & 1 & 1 & 0 & 1 & 0 \\ 2 & 1 & 1 & 0 & 0 & 1 \\ 3 & 1 & 1 & 0 & -1 & -1 \\ 4 & 1 & 0 & 1 & 1 & 0 \\ 5 & 1 & 0 & 1 & 0 & 1 \\ 6 & 1 & 0 & 1 & -1 & -1 \\ 7 & 1 & -1 & -1 & 1 & 0 \\ 8 & 1 & -1 & -1 & 0 & 1 \\ 9 & 1 & -1 & -1 & -1 & -1\end{array}$

Figure 7. Designmatrix used for the Initial First-order Configural Frequency Analysis for the Data from Kieser and Victor (1999), p.969.

into the R-console. Executing this command results in the (abbreviated) output on the screen depicted in Figure 7.

It is obvious that the design matrix used for calculations matches the one depicted in matrix (22), except for the last column which is missing. In our next analyses we want to blank out the last configuration ' 33 ' of the nine possible configurations; we therefore modify the design matrix accordingly. Hence, we add an additional column (vector) with the length of nine entries which contains only zeros except for the last entry. The last entry is a ' 1 ' to blank out the respective configuration ' 33 '. In order to do so, we just type in the following commands in Listing 7 , which will modify the design matrix and store it in an R-object called 'des_01' (line number 1 in Listing 7) and that in turn will be displayed on the screen (line number 2 in Listing 7).

Listing 7. $\mathrm{R}$ commands to blank out the last data cell for the functional configural frequency analysis example.

1. des_01<-as .matrix (cbind (resd4\$designmatrix, blank $=\mathbf{c}(0,0,0,0,0,0,0,0,1)))$

2. des_01

3. resd4_01<-CFA $(\mathrm{d} 4$, form $=$ des_01)

4. $\quad$ summary (resd4_01, showall = TRUE)

To run the functional configural frequency analysis with the blanked out cell ' 33 ', we assign the newly modified design matrix simply to the 'form' argument of the function CFA (). The command in line number 3 in listing 7 will assign the results of the functional configural frequency analysis to an object named 'resd4_01'. The command in line number 4 in listing 7 will summarize it on the screen by using the summary () function.

Inspecting the (abbreviated) results depicted in Figure 8, one can clearly see that the significant configurations of the firstorder configural frequency analysis vanished, which suggests quasi-independence in the presence of one type - that is, the blanked out cell ' 33 '. As a reminder the blanked out cell is marked with the character ' $\mathrm{b}$ ' in the column 'Type' in the output (see last line in Figure 8).

A closer look at the information theoretical criteria AIC and BIC for the functional approach in comparison to the former ordinary main effects model reveals that the model of the functional configural frequency analysis approach results in a better overall model fit! Therefore, if there is concern that the assumption of independence does not apply to the entire contingency table, because of extreme outlying cells, the functional configural frequency analysis approach can be used to test for quasi-independence after blanking out suspicious cells.

\section{Summary}

Configural frequency analysis can be seen as a useful tool for the analysis of categorical or categorized data. The developmental theory underpinning this view assumes that humans are seen as inseparable units, embedded in their contexts but who try to be active producers of their development. Configural frequency analysis and LLM are related statistical tools. They calculate the expected frequencies in the same manner. But configural frequency analysis and LLM do not overlap completely. There are log-linear models that cannot be used as base models for configural frequency analysis. For many research questions, however, LLM and configural frequency analysis can be used as complimentary statistical tools. LLM looks for models with appropriate goodness-of-fit; that is, the corresponding Chisquare and LR-values result in $p$-values greater than a previously chosen level of significance ( $\alpha$ is usually set to $\alpha=.05$ ). An inspection of the residuals reveals deviations from an assumed base model. The residuals indicate whether they were caused by chance, artifacts, or other errors in the data. The residuals also indicate whether the model itself needs to be modified. Configural frequency analysis needs a non-fitting model $(p<.05)$ in order to detect types and/or antitypes. The expected frequencies are calculated according to any underlying null model which is specified in the design matrix using the GLM approach. Configural frequency analysis and LLM pursue different goals: configural frequency analysis is "cell-oriented" and LLM is "dependency-structure oriented" (Victor, 1989). In most cases first-order configural frequency analysis is applied, if one is interested in multivariate patterns or configurations. Prediction configural frequency analysis is suitable for longitudinal data, where the criterion is measured after the predictors. First-order configural frequency analysis is symmetrical, treating all variables as equal. In contrast prediction configural frequency analysis defines variables as independent and dependent. If there are significant interactions between the independent and dependent variables, this suggests a more directed relationship between the two sets of variables. Two-group configural frequency analysis is an appropriate tool, for instance, in evaluation research. One can compare the occurrence of types or antitypes between two samples or groups. When, for example, the desired configurations are significant in the experimental group but not in the control group, the intervention seems to be successful. Two-group configural frequency analysis can also be applied for repeated measures analyses. Configural frequency analysis has its limits if there are too many zero cells. Sparse data are a problem that cannot be overcome easily, one may switch to smaller cross-tables or collect more data, if possible. In order to detect extreme outlier cells or so-called Victor cells one should apply the functional configural frequency analysis approach. By blanking out extreme cells with the help of a selfdesigned design matrix one can test for quasi-independence of the contingency table in the presence of one or more types. In short, the use of the person-centered approach broadens one 


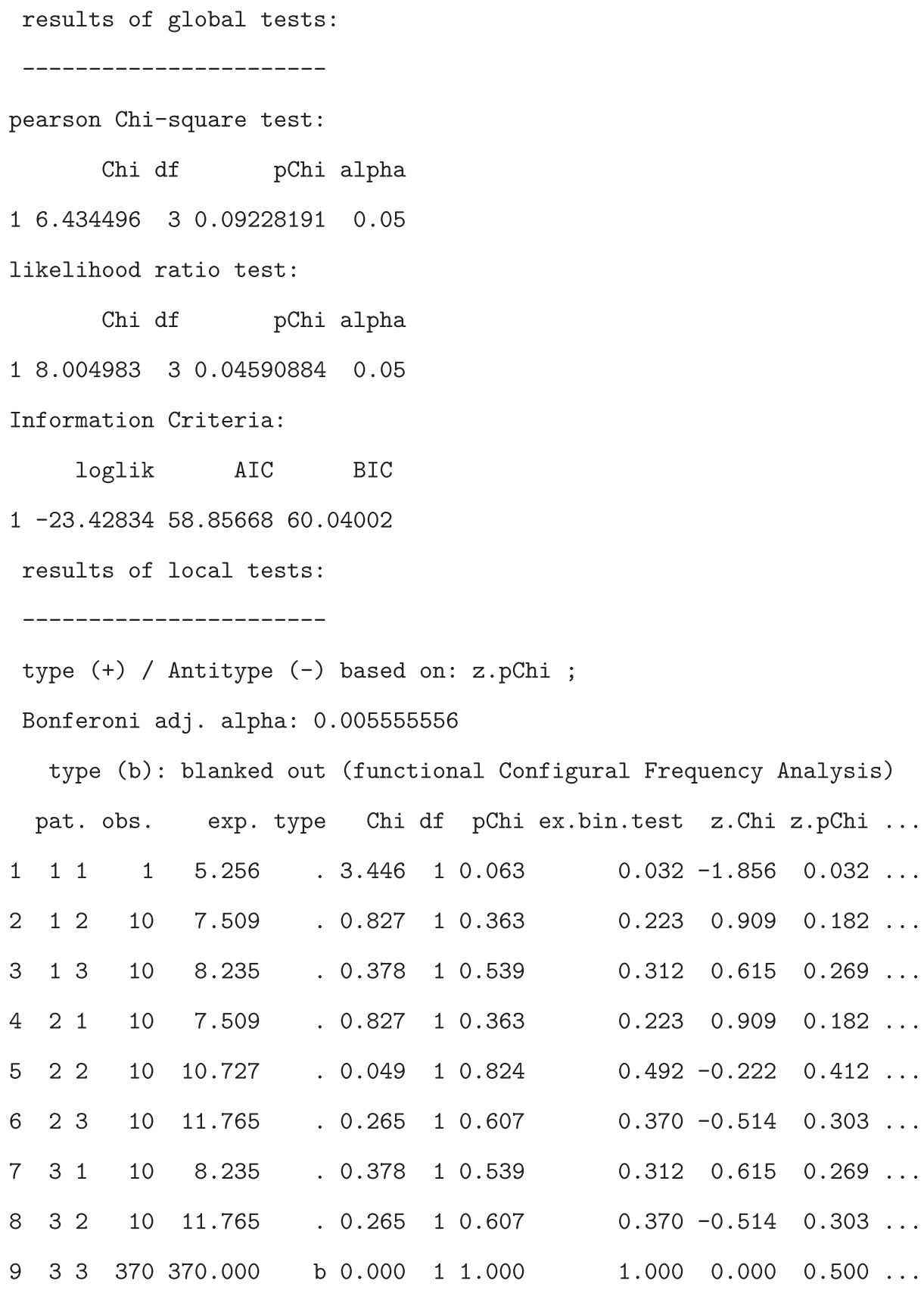

Figure 8. R-console Output of the Results for a Functional Configural Frequency Analysis for the Data from Kieser and Victor (1999), p.969.

view and enriches our research opportunities in developmental psychology.

From a statistical point of view the person-centered approach has a number of distinctive features:

one looks for persons or units with characteristic patterns or configurations;

configural frequency analysis has few requirements with regard to sample size, however, large contingency tables together with sparse data can be problematic;

the underlying sampling distribution is the multinomial distribution, instead of the normal distribution;

instead of a linear combination (e.g. equation 23)

$$
y=b_{0}+b_{1} X_{1}+b_{2} X_{2}+e
$$

we are dealing with a multiplicative relationship, which can be transformed into an additive relationship through the logarithm as in equation (24)

$$
\ln e_{i j}=\lambda_{0}+\lambda_{i} A_{i}+\lambda_{j} B_{j}
$$

Configural frequency analysis belongs to the non-parametric method, although parametric versions of configural frequency analysis have been specified (Spiel \& von Eye, 1993).

Therefore, configural frequency analysis is a very useful personcentered approach. One may specify an underlying null model that corresponds to first-order configural frequency analysis, prediction configural frequency analysis or two-group configural frequency analysis. As an alternative, the outlier cells can be treated as 
structural zeros, using functional configural frequency analysis. While in the past there were only a few self-made freeware programs for the analysis of configural frequency analysis, the newly developed R-package confreq is a versatile tool for computational statistics. It is welcomed by the growing R-community and its advantages include no costs and compatibility with LTEX.

\section{Funding}

The author(s) received no financial support for the research, authorship, and/or publication of this article.

\section{Supplemental material}

Supplementary material for this article is available online.

\section{References}

Agresti, A. (2013). Categorical data analysis - 3 rd edition. Hoboken, NJ: John Wiley and Sons, Inc.

Alexandrowicz, R. W. (2013). $R$ in 10 Schritten: Einführung in die statistische Programmierumgebung [R in 10 steps: Introduction to the statistical computing environment] (1st ed.). Stuttgart, Germany: UTB GmbH.

Angerheofer, U., Kullik, U., \& Masendorf, F. (1992). Denk- und Rechenförderung lernbeeinträchtigter Kinder: Multivariate Änderungsbeurteilung mittels Prädiktions-KFA [Reasoning and mathemathics training for learning disabled children: A multivariate rating of change based on prediction-CFA]. Psychologie in Erziehung und Unterricht [Psychology in Parental Guidance and Education], 39, 190-195.

Belsky, J., \& Pluess, M. (2009). The nature (and nurture?) of plasticity in early human development. Perspectives on Psychological Science, 4 (4), 345-351.

Bergman, L. R., \& Magnusson, D. (1997). A person-oriented approach in research on developmental psychopathology. Development and Psychopathology, 9 (2), 291-319.

Bergman, L. R., Magnusson, D., \& Khouri, B. M. (2003). Studying individual development in interindividual context: A person-oriented approach. East Sussex, UK: Psychology Press.

Bergman, L. R., von Eye, A., \& Magnusson, D. (2006). Person-oriented research strategies in developmental psychopathology. In D. Cicchetti \& D. Cohen (Eds.), Developmental Psychopathology (2nd ed., pp. 850-888). London, UK: Wiley.

Gutiérrez-Peña, E. (2012). Bayesian predictive configural frequency analysis. Psychological Test and Assessment Modeling, 54 (3), 285.

Gutiérrez-Peña, E., \& von Eye, A. (2000). A Bayesian approach to configural frequency analysis. Journal of Mathematical Sociology, $24,151-175$.

Heine, J.-H. (2015). confreq: Configural frequencies analysis using log-linear modeling (V 1.4). Retrieved from http://cran.r-project. org/web/packages/confreq/index.html (R package version 1.4)

Holland, B. S., \& Copenhaver, M. D. (1987). An improved sequentially rejective Bonferroni test procedure. Biometrics, 43, 417-423.

Hommel, G. (1988). A stagewise rejective multiple test procedure based on a modified Bonferroni test. Biometrika, 75, 383-386.

Kieser, M., \& Victor, N. (1991). A test procedure for an alternative approach to configural frequency analysis. Methodika, 5, 87-97.

Kieser, M., \& Victor, N. (1999). Configural frequency analysis CFA revisited - A new look at an old approach. Biometrical Journal, 41, 967-983.

Klauer, K. (1989). Denktraining für Kinder I. Ein Programm zur intellektuellen Förderung [A reasoning training for children: A program for the enhancement of intellectual abilities]. Göttingen, Germany: Hogrefe.

Krauth, J., \& Lienert, G. A. (1973). Die Konfigurationsfrequenzanalyse (KFA) und ihre Anwendung in Psychologie und Medizin: ein multivariates nichtparametrisches Verfahren zur Aufdeckung von Typen und Syndromen [Configural frequency analysis and its application in psychology and medicine]. Freiburg; München, Germany: Alber Karl.

Langeheine, R. (1980). Log-lineare Modelle zur multivariaten Analyse qualitativer Daten: eine Einführung [Log-linear models for the analysis of qualitative data: An introduction]. München, Germany: R. Oldenbourg Verlag.

Lehmacher, W. (2000). Die Konfigurationsfrequenzanalyse als Komplement des log-linearen Modells [Configural Frequency Analysis as a complimentary tool to log-linear modeling]. Psychology Science, 42 (3), 418-427.

Lerner, R. M., \& Busch-Rossnagel, N. A. (1981). Individuals as producers of their development: A life-span perspective. New York: Academic Press.

Lösel, F., Beelmann, A., Stemmler, M., \& Jaursch, S. (2006). Prävention von Problemen des Sozialverhaltens im Vorschulalter [Prevention of behavioral problems in kindergarten]. Zeitschrift für Klinische Psychologie und Psychotherapie [Journal of Clinical Psychology and Therapy], 35 (2), 127-139.

MacCallum, R. C., Zhang, S., Preacher, K. J., \& Rucker, D. D. (2002). On the practice of dichotomization of quantitative variables. Psychological methods, 7 (1), 19.

Magnusson, D., \& Allen, V. E. (1983). Human development: An interactional perspective. New York: Academic Press.

Magnusson, D., \& Mahoney, J. L. (2001). Reports from the project Individual Development and Adaptation. A holistic person approach for research on positive development (Tech. Rep. No. 76). Stockholm, Sweden: University of Stockholm: Department of Psychology.

McArdle, J. J. (2001). A latent difference score approach to longitudinal dynamic structural analysis. In R. Cudeck, S. du Toit, \& D. Sorbom (Eds.), Structural equation modeling: Present and future (pp. 342-380). Lincolnwood, Il.: Scientific Software International.

R Development Core Team. (2015). R: A Language and Environment for Statistical Computing. Vienna, Austria. Retrieved from http:// www.R-project.org/

Raine, A., Venables, P. H., \& Mednick, S. A. (1997). Low resting heart rate at age 3 years predisposes to aggression at age 11 years: Evidence from the Mauritius Child Health Project. Journal of the American Academy of Child and Adolescent Psychiatry, 36 (10), $1457-1464$.

Silbereisen, R., \& Noack, P. (2006). Kontexte und Entwicklung [Contexts and development]. In Enzyklopädie der Psychologie [Encyclopedia of psychology]. Vols. 1 Theorien, Modelle und Methoden der Entwicklungspsychologie [Theories, models, and methods of developmental psychology] (pp. 311-368). Göttingen, Germany: Hogrefe.

Spiel, C., \& von Eye, A. (1993). Configural frequency analysis as a parametric method for the search of types and antitypes. Biometrical Journal, 35 (2), 151-164.

Stemmler, M. (2014). Person-Centered Methods. Cham, Germany: Springer International Publishing.

Stemmler, M., \& Bingham, C. R. (2004). Nonparametric testing of improvement scores in a two sample prepost design. Psychology Science, 39, 208-216. 
Stemmler, M., \& Hammond, S. (1997). Configural frequency analysis of dependent samples for intra-patient treatment comparisons. Studia Psychologica, 39, 167-175.

Stemmler, M., Lautsch, E., \& Martinke, D. (Eds.). (2008). Configural frequency analysis and other non-parametrical methods: A Gustav A. Lienert memorial issue. Lengerich, Germany: Pabst Publishing.

Stemmler, M., \& Lösel, F. (2012). The stability of externalizing behavior in boys from preschool age to adolescence: A person-oriented analysis. Psychological Test and Assessment Modeling, 54 (2), 195.

Stemmler, M., Lösel, F., Beelmann, A., Jaursch, S., \& Zenkert, B. (2005). Child problem behavior in kindergarten and in primary school: A comparison between prediction configural frequency analysis and multiple regression. Psychology Science, 47 (3/4), 467-478.

Stemmler, M., \& Masendorf, F. (1998). Konfigurale Auswertung von Daten aus Prätest-Posttest Kontrollgruppen Designs [Configural data analysis based on pretest-posttest control group designs]. Zeitschrift für Klinische Psychologie und Psychotherapie [Journal of Clinical Psychology and Psychotherapy], 46, 267-276.

Stemmler, M., \& von Eye, A. (2012). Configural frequency analysis (CFA) and other non-parametrical statistical methods: Introduction to Special Topic (Part II). Psychological Test and Assessment Modeling, 54 (3), 284.

Victor, N. (1983). A note on contingency tables with one structural zero. Biometrical Journal, 25, 283-289.

Victor, N. (1989). An alternative approach to configural frequency analysis. Methodika, 3, 61-73.

von Eye, A. (1990). Introduction to configural frequency analysis: The search for tpes and antitypes in cross-classifications. Cambridge, UK: Cambridge University Press. von Eye, A. (2002). Configural frequency analysis: Methods, models, and applications. Mahwah, NJ: Lawrence Erlbaum Associates.

von Eye, A., Bergman, L. R., \& Hsieh, C.-A. (2015). Person-oriented approaches in developmental science. In W. F. Overton \& P. Molenaar (Eds.), Handbook of child psychology and developmental science - theory and methods (pp. 789-841). Hoboken, NJ: Wiley.

von Eye, A., \& Gutiérrez-Peña, E. (2004). Configural frequency analysis: the search for extreme cells. Journal of Applied Statistics, 31 (8), 981-997.

von Eye, A., Lerner, R. M., \& Lerner, J. V., \& Bowers. (2012). The development of delinquent behavior: Variable - and person-oriented methods of analysis. In T. Bliesener, A. Beelmann, \& M. Stemmler (Eds.), Antisocial behavior and crime: Contributions of developmental and evaluation research to prevention and intervention (pp. 293-314). Toronto: Hogrefe Publishing.

von Eye, A., \& Mair, P. (2001). A functional approach to configural frequency analysis (Tech. Rep. No. 48). Vienna, Austria: WU Vienna University of Economics and Business: Department of Statistics and Mathematics.

von Eye, A., Mair, P., \& Eun-Young, M. (2010). Advances in configural frequency analysis. New York: The Guilford Press. von Eye, A., \& Mun, E.-Y. (2013). Log-linear modelling: Concepts, interpretation, and application. Hoboken, NJ: John Wiley and Sons, Inc.

Wermuth, N. (1973). Anmerkungen zur Konfigurationsfrequenzanalyse [Notes on configural frequency analysis]. Zeitschrift für Klinische Psychologie und Psychotherapie [Journal of Clinical Psychology and Therapy], 3, 5-21.

Wickens, T. D. (1989). Multiway contingency tables analysis for the social sciences. Hillsdale, NJ: Lawrence Erlbaum Associates. 